\title{
Recria de cordeiras em pastagem nativa melhorada submetida à fertilização nitrogenada. 2. Produção animal
}

\author{
Igor Justin Carassai ${ }^{1}$, Carlos Nabinger ${ }^{2}$, Paulo César de Faccio Carvalho ${ }^{2}$, Davi Teixeira dos \\ Santos ${ }^{1}$, Fabiana Kellermann de Freitas ${ }^{1}$, Thais Devincenzi ${ }^{3}$, Raquel Rolim Cardoso ${ }^{3}$ \\ 1 Programa de Pós-graduação em Zootecnia - Universidade Federal do Rio Grande do Sul (UFRGS). \\ ${ }^{2}$ Departamento de Plantas Forrageiras e Agrometeorologia, Faculdade de Agronomia, UFRGS, Av. Bento Gonçalves, $n^{\circ} 7.712$, \\ CEP: 91501-970, Porto Alegre, RS \\ 3 Curso de Graduação em Agronomia, UFRGS
}

RESUMO - Avaliou-se o desempenho de cordeiras de corte para o acasalamento no outono em pastagem nativa melhorada fertilizada com adubo mineral fórmula 5-20-20 (250 kg/ha). Utilizaram-se duas doses de nitrogênio (100 e $200 \mathrm{~kg} / \mathrm{ha}$ na forma de uréia), fracionadas em duas aplicações (70\% em 3 de fevereiro e 30\% em 17 de março de 2005) considerando controle piquetes sem aplicação de nitrogênio. Os tratamentos foram distribuídos em blocos completos com duas repetições. O método de pastejo foi contínuo com lotação variável e oferta de forragem de $16 \%$ (16 kg MS/100 kg peso vivo [PV]). O período de avaliação foi de 21 de janeiro a 31 de maio. As variáveis estudadas foram taxa de lotação, carga animal, desaparecimento de MS em \%PV, ganho de peso médio diário, escore de condição corporal, ganho de peso por área e taxa de prenhez. O desaparecimento de MS em \%PV não diferiu entre as doses de nitrogênio e os períodos de avaliação e apresentou valores limitantes em relação ao provável consumo potencial. As doses de nitrogênio tiveram efeito positivo sobre a taxa de lotação e a carga animal, que não diferiram no decorrer das avaliações. O escore de condição corporal, o ganho médio diário e o ganho de peso por área apresentaram variações significativas entre os períodos de avaliação, como resultado da influência do déficit hídrico registrado durante o período experimental. A taxa de prenhez não foi influenciada pelas doses de nitrogênio. Apesar do déficit hídrico, que comprometeu negativamente as variáveis produtivas da pastagem e a resposta animal às doses de nitrogênio aplicadas, a pastagem nativa melhorada por meio de adubação pode suportar elevadas taxas de lotação.

Palavras-chave: ganho de peso médio diário, ganho de peso por área, oferta de forragem, pastejo contínuo, prenhez

\section{Rearing of lambs in improved native pasture submitted to nitrogen fertilization. 2. Animal production}

ABSTRACT - The performance of lambs for the mating in the autumn in improved native pasture fertilized with $250 \mathrm{~kg} / \mathrm{ha}$ of mineral formula 5-20-20 was evaluated. Two levels of nitrogen (N) (100 and $200 \mathrm{~kg} / \mathrm{ha}$ of $\mathrm{N}$ as urea) divided in two applications, 70\% and 30\%, in February, 3, and March, 17, 2005, respectively, were tested in a complete block design with two replications. Plots without $\mathrm{N}$ application were used as control. Evaluation was done on continuous variable stocking in order to maintain $16 \%$ of forage allowance $(16 \mathrm{~kg} \mathrm{DM} / 100 \mathrm{~kg}$ live weight [LW]). Grazing period extended from January, 21 to May, 31. Studied variables were: stocking rate, DM disappearance, in \% of LW, average daily gain, body score condition, weight gain per area and, pregnancy rate. The DM disappearance did not differ between treatments and periods of evaluation, presenting limited values in relation to the probable potential intake. The stocking rate showed positive effect of the level of $\mathrm{N}$ and did not differ along the evaluations. The other studied variables (body score condition, average daily gain and weight gain per area) presented significant variations between evaluation periods due to influence of the water deficit that occurred during the experimental period. The pregnancy rate did not show significant effect of the level of N. Despite the water deficit which occurred during the experimental period and compromised the performance of the pasture variables and, by consequence, animal response to nitrogen fertilization, the native pasture improvement by fertilization is capable to support high stocking rates.

Key Words: average daily weight gain, continuous stocking, forage allowance, live weight gain per area, pregnancy

\section{Introdução}

A competitividade econômica tem exercido fortes pressões e conduzido a diminuição da área de pastagem nativa, substituída por culturas de maior retorno financeiro em curto prazo. A reconhecida limitação que a fertilidade da maioria dos solos impõe à expressão do potencial da pastagem nativa torna necessária a busca pelo conhecimento 
das respostas da pastagem à fertilização, que responde à adubação como qualquer outro tipo de pastagem, conforme atestaram diversos autores (Barcellos et al., 1987; Bemhaja et al., 1998; Boggiano et al., 2000; Gomes, 2000; Guma, 2005).

A ovinocultura pode proporcionar aumento da escala de produção e agregação de valor ao produto comercializado. Entretanto, são necessários estudos visando à intensificar a exploração de forma sustentável. Neste sentido, torna-se fundamental definir o potencial de produção da pastagem nativa utilizada com ovinos, principalmente relacionado aos aspectos produtivos e reprodutivos, sobretudo animais jovens, tendo em vista o baixo desempenho desta categoria no rebanho gaúcho, que são normalmente acasaladas aos 18-20 meses de idade.

A produtividade de uma ovelha depende de vários fatores, como a idade ao atingir a puberdade e a capacidade de reprodução. Quanto mais precocemente estas características forem atingidas, maior a taxa de prenhez e maior o número de cordeiros produzidos, o que aumenta a eficiência de produção (Roda et al., 1993; Lewis et al., 1996; Powell et al., 1996).

Fêmeas ovinas podem se reproduzir ao atingir a puberdade, quando aparecem os primeiros cios, entretanto, nesta fase, a maturidade sexual ainda não foi atingida, uma vez que o animal não expressa seu ótimo desempenho reprodutivo. A decisão de colocar um animal jovem em reprodução tem grande importância por afetar seu desempenho reprodutivo futuro (Sá et al., 1998). A subfertilidade em cordeiras é uma das causas de redução da produtividade do rebanho. Embora a taxa de fertilidade sofra influência de vários fatores, 20 a $40 \%$ das cordeiras falham em produzir seu primeiro cordeiro (Davies \& Beck, 1993; Beck \& Davies, 1994; Beck et al., 1996).

Avaliou-se neste trabalho o desempenho de cordeiras destinadas à reprodução ao final do outono criadas em pastagem nativa melhorada adubada com nitrogênio.

\section{Material e Métodos}

O experimento foi realizado em uma área de pastagem nativa melhorada da Estação Experimental Agronômica (EEA) da Universidade Federal do Rio Grande do Sul (UFRGS), situada no km 146 da BR - 290, município de Eldorado do Sul - RS (3005'52" S, 51³9'08" W e altitude média de $46 \mathrm{~m}$ ) compreendendo a região fisiográfica da Depressão Central. A área experimental apresenta solo classificado como Argissolo Vermelho Distrófico (EMBRAPA, 1999), profundo, bem drenado, de textura arenosa a franco-argilosa. O clima da região é o Cfa (subtropical úmido) com verão quente, segundo a classificação de Köppen (Moreno, 1961). De acordo com Bergamaschi et al. (2003), a precipitação total média anual naEEA da UFRGS situa-se em torno de $1.440 \mathrm{~mm}$, com média mensal de $120 \mathrm{~mm}$. Os eventos meteorológicos registrados durante o período experimental (Figura 1) foram obtidos na Estação Meteorológica do Departamento de Plantas Forrageiras e Agrometeorologia/UFRGS, localizada a cerca de $400 \mathrm{~m}$ da área experimental.

A pastagem nativa onde foi realizado o experimento é caracterizada como de sucessão secundária. Mella (1980) realizou experimento de consorciação de Paspalum guenoarum e Desmodium intortum, mas estas espécies não persistiram e houve o retorno da vegetação natural (sucessão secundária), caracterizada por Rosito (1983). Em 1996, a área foi redimensionada, o solo foi novamente corrigido (3 t/ha calcário e 500 kg/ha 5-20-20) e iniciaram-se trabalhos com bovinos em pastejo (Boggiano, 2000; Gomes, 2000; Guma, 2005) e aplicação anual de nitrogênio $(0,100$ e $200 \mathrm{~kg} / \mathrm{ha}$ de $\mathrm{N})$.

A área experimental foi composta de seis piquetes (unidades experimentais), com área média de 0,52 ha, totalizando 3,12 ha. A área foi roçada em 31 de agosto e 29 de novembro de 2004. A pastagem foi adubada em 10 de novembro com $250 \mathrm{~kg} / \mathrm{ha}$ de adubo mineral de fórmula 5-20-20. Avaliaram-se os desempenhos produtivo e reprodutivo de cordeiras em pastejo em resposta ao efeito da aplicação de duas doses de nitrogênio (N) (100 e $200 \mathrm{~kg} /$ ha de $\mathrm{N}$, na forma de uréia) fracionado em duas vezes: $70 \%$ em 3 de fevereiro e $30 \%$ em 17 de março de 2005 , tendo como controle piquetes sem aplicação de nitrogênio.

O pastejo foi realizado em lotação contínua com carga variável, utilizando-se nove animais-teste por unidade experimental e um número variável de animais reguladores (Mott \& Lucas, 1952). A oferta de forragem total (OF) pretendida foi de $16 \%$ (16 kg MS/100 kg peso vivo [PV]/dia) em todos os níveis de nitrogênio. Segundo Gibb \& Treacher (1975), deve-se ofertar no mínimo três vezes o potencial de consumo desses animais, que é de 3,5\% PV (NRC, 1985) para que a ingestão de MS não esteja limitada. O período de avaliação foi de 21/1/2005 a 31/5/2005, totalizando 131 dias (110 dias no pré-acasalamento, 6 dias de preparo para o acasalamento e 15 dias de acasalamento). Foram utilizadas cordeiras da raça Suffolk com seis meses de idade e peso médio inicial de $38,4 \mathrm{~kg}$. As pesagens dos animais foram realizadas em intervalo de 28 dias, respeitando-se jejum prévio de 12 horas em todas as pesagens. Por ocasião de cada pesagem, avaliou-se também o escore de condição corporal (ECC) de cada animal pela técnica descrita por 
Russel (1991), que consiste em palpar a rugosidade dos processos transversos e dorsais das vértebras lombares avaliando a deposição de gordura e o desenvolvimento muscular e atribuindo escores de 1 (muito magro) a 5 (muito gordo).

O controle de endoparasitas foi realizado por meio de dosificações dos animais com nitroxinil e moxidectina, quando apresentavam infestação superior a 500 ovos por grama de fezes. Os animais receberam sal mineral à vontade, e tinham acesso a abrigos com sombra.

A massa de forragem instantânea (MF, em kg/ha de MS) foi avaliada a cada 28 dias, utilizando-se o método comparativo, empregando a "dupla amostragem" (Haydock \& Shaw, 1975) e realizando-se 30 estimativas visuais em quadrados de $0,25 \mathrm{~m}^{2}$ por piquete (unidade experimental). Para determinação do material morto (MM, em \%) foram separados manualmente o material verde e o material morto dos cortes de fora de gaiola. Por meio da subtração do material morto (MM) da massa de forragem, obteve-se a massa de forragem verde (MFV, em kg/ha de MSV). Para avaliação da taxa de acúmulo diário de MS (TAD, em kg/ha de MS), utilizou-se a técnica do triplo emparelhamento (Moraes et al., 1990). Foram utilizadas três gaiolas de exclusão ao pastejo por piquete (repetição). A TAD foi obtida pela diferença entre a massa de forragem de dentro da gaiola na medição i e a massa de forragem de fora da gaiola da medição i - 1. A taxa de desaparecimento diário de MS (TDD, em $\mathrm{kg} / \mathrm{ha}$ de MS) foi obtida pela diferença entre a massa de forragem de dentro da gaiola e a massa de forragem de fora da gaiola da mesma avaliação.

Com a massa de forragem, a massa de forragem verde, a TAD e a carga animal (CA, em kg/ha de PV) utilizada em cada período de avaliação, calcularam-se a oferta de forragem total (OF) e a oferta de forragem verde de MS (OFV, em $\mathrm{kg} \mathrm{MSV/100} \mathrm{kg} \mathrm{PV/dia),} \mathrm{por} \mathrm{meio} \mathrm{da} \mathrm{seguinte} \mathrm{fórmula:}$

$$
\mathrm{OF}=(\mathrm{MF} / \mathrm{n}+\mathrm{TAD}) \times 100 / \mathrm{CA}
$$

em que $\mathrm{OF}=$ oferta de forragem $(\% \mathrm{PV}) ; \mathrm{MF}=$ massa de forragem média ( $\mathrm{kg} / \mathrm{ha}$ de $\mathrm{MS}) ; \mathrm{n}=$ número de dias do ciclo de pastejo (dias); TAD = taxa de acúmulo diário de MS $(\mathrm{kg} / \mathrm{ha}$ de $\mathrm{MS}) ; \mathrm{CA}=$ carga animal média do ciclo de pastejo (kg/ha de PV).

O ganho médio diário (GMD, em kg/animal) dos animais foi obtido pela diferença entre os pesos final e inicial dos animais-teste, em cada período experimental, dividida pelo número de dias do período. A carga animal média (CA, em $\mathrm{kg} /$ ha de PV) foi calculada, para cada unidade experimental, pela adição do peso médio dos animais-teste, com o peso médio de cada animal regulador, multiplicado pelo número de dias que esses animais permaneceram na pastagem, dividido pelo número total de dias de pastejo. A divisão da CA pelo peso médio dos animais teste forneceu a taxa de lotação média (LOT, em animais/ha). O ganho de peso por área (GPA, em kg/ha de PV) foi obtido pela multiplicação LOT pelo GMD dos animais-teste e pelo número de dias de pastejo. O desaparecimento de MS em \%PV (DPV, em \% do PV) foi obtido dividindo-se TDD pela LOT e, depois, o resultado foi dividido pelo peso médio dos animais-teste.

As cordeiras foram acasaladas a partir da segunda quinzena de maio, por 15 dias, e por meio de monta natural. A porcentagem de carneiros no período de monta foi semelhante entre os níveis de nitrogênio ( $\mathrm{P}=0,0919), 10 \%$ na média, valor recomendado quando se utiliza a técnica de sincronização de estro (Moraes et al., 2002).

Foram utilizados carneiros da raça Suffolk com dois dentes permanentes e peso médio de $67 \mathrm{~kg}$, previamente submetidos a exame clínico. $\mathrm{O}$ exame andrológico não foi realizado e, por isso, realizou-se um rodízio dos carneiros dentro de cada bloco experimental, trocando-os de piquete a cada dois dias na tentativa de eliminar qualquer efeito negativo que algum reprodutor poderia ocasionar. Utilizou-se a técnica de sincronização de estros com prostaglandina ( $\mathrm{PG}_{2} \alpha$, Dinoprost Trometamina), realizando-se o seguinte protocolo: dia 1 - entrada dos carneiros nos piquetes; dia 2 - primeira sincronização; dias 3, 4, 5, 6, 7 e 8 -observação de estro; dia 9 - segunda sincronização; dias 10, 11, 12, 13,14 e 15 - observação de estro. A observação de estro consistiu da verificação das cordeiras montadas (marcadas com tinta na região da garupa) a cada dia da estação de acasalamento. Para isso, utilizou-se uma mistura de tinta e graxa, que foi aplicada no peito dos carneiros na data de sua entrada nos piquetes. Foram sincronizadas apenas as cordeiras que ainda não tinham sido montadas.

Aproximadamente aos 50 dias após a retirada dos carneiros, foi realizado o diagnóstico de gestação, por ultrasonografia utilizando-se um aparelho VetScan 2, equipado com transdutor setorial de 3,5 Mhz. As cordeiras foram examinadas sem jejum prévio, usando-se um brete a $40 \mathrm{~cm}$ do solo, com o exame realizado na região inguinal direita do animal. A taxa de prenhez (TP, em \%) foi calculada conforme descrito por Ribeiro et al. (2002).

O delineamento experimental foi de blocos completos casualizados com duas repetições. Os resultados foram submetidos à análise de variância e ao teste $\mathrm{F}$ a $10 \%$ de significância, e as médias comparadas pelo teste Tukey no mesmo nível. Também foram realizadas análise de regressão até terceira ordem entre doses de nitrogênio e período de avaliação, considerando-se, para cada período, o número de dias decorridos desde o início do experimento ( $\mathrm{x}=$ dia 1 a 110). As variáveis reprodutivas (sincronização e PP) foram submetidas ao teste do qui-quadrado $\left(\chi^{2}\right)$ 
$(\mathrm{P}<0,10)$. As análises foram realizadas utilizando-se o aplicativo computacional SAS (1997). As variáveis TAD, GMD e GPA foram acrescidas de uma constante, em virtude da presença de valores negativos para fins de ajuste do coeficiente de variação (CV) dos dados para a execução do programa estatístico.

$\mathrm{O}$ modelo matemático geral referente à análise das variáveis estudadas foi representado por:

$$
\mathrm{Yikj}=\mu+\mathrm{Bi}+\mathrm{Ti}+\mathrm{Pk}+\mathrm{TPik}+\mathrm{Eij}
$$

em que Yikj = variáveis dependentes; $\mu=$ média de todas as observações; $\mathrm{Bi}=$ efeito do bloco $\mathrm{i} ; \mathrm{Ti}=$ efeito do nível de nitrogênio i; $\mathrm{Pk}=$ efeito do período $\mathrm{k}$; TPik = interação nível de nitrogênio $\mathrm{i} \times$ período $\mathrm{k} ; \mathrm{Eij}=$ erro aleatório associado a cada observação j.

\section{Resultados e Discussão}

Os dados do balanço hídrico climático da série histórica 1969-1999 e do período experimental foram calculados pelo método de Thornthwaite \& Mather (1955) para $75 \mathrm{~mm}$ de capacidade de campo (Figura 1). Nos meses de janeiro a março de 2005, a precipitação foi muito inferior à média histórica, causando déficit hídrico em grande parte do período de avaliação. O déficit hídrico acumulado durante o período experimental foi de $237,5 \mathrm{~mm}$, enquanto o esperado de acordo com a média histórica seria apenas $56,5 \mathrm{~mm}$. Assim, em virtude da falta de água, durante três meses a pastagem esteve sob condições extremamente limitantes para o seu desenvolvimento.

Os dados de massa de forragem ajustaram-se ao modelo de regressão linear $(\hat{\mathrm{Y}}=-12,67371 \mathrm{x}+3.852,886$; $\left.\mathrm{R}^{2}=0,6048 ; \mathrm{P}<0,0001\right)$ e decresceram de $12,6 \mathrm{~kg} / \mathrm{ha}$ de $\mathrm{MS}$ a cada dia de pastejo. Os maiores valores de MF $(3.515 \mathrm{~kg} / \mathrm{ha}$ de MS) (Tabela 1) no primeiro período decorreram do fato de a área ter sido excluída do pastejo antes do início das avaliações. Os valores médios para os níveis 0,100 e $200 \mathrm{~kg} / \mathrm{ha}$ de nitrogênio foram, respectivamente, 2.678, 3.018 e $3.220 \mathrm{~kg} / \mathrm{ha}$ de MS e foram maiores $(\mathrm{P}=0,0026) \mathrm{com}$ a adubação nitrogenada nos níveis 100 e $200 \mathrm{~kg} / \mathrm{ha}$ de nitrogênio, que não diferiram entre si. $\mathrm{O}$ valor médio de massa de forragem ao longo do período experimental ( $2.972 \mathrm{~kg} / \mathrm{ha}$ de MS) não indica limitação de consumo para os animais. No entanto, como conseqüência do déficit hídrico, a massa de forragem verde tornou-se muito baixa nos períodos intermediários e foi significativamente inferior à dos períodos inicial e final.

Em razão do déficit hídrico, a MFV apresentou nos períodos intermediários seus menores valores $(\mathrm{P}=0,0002)$,
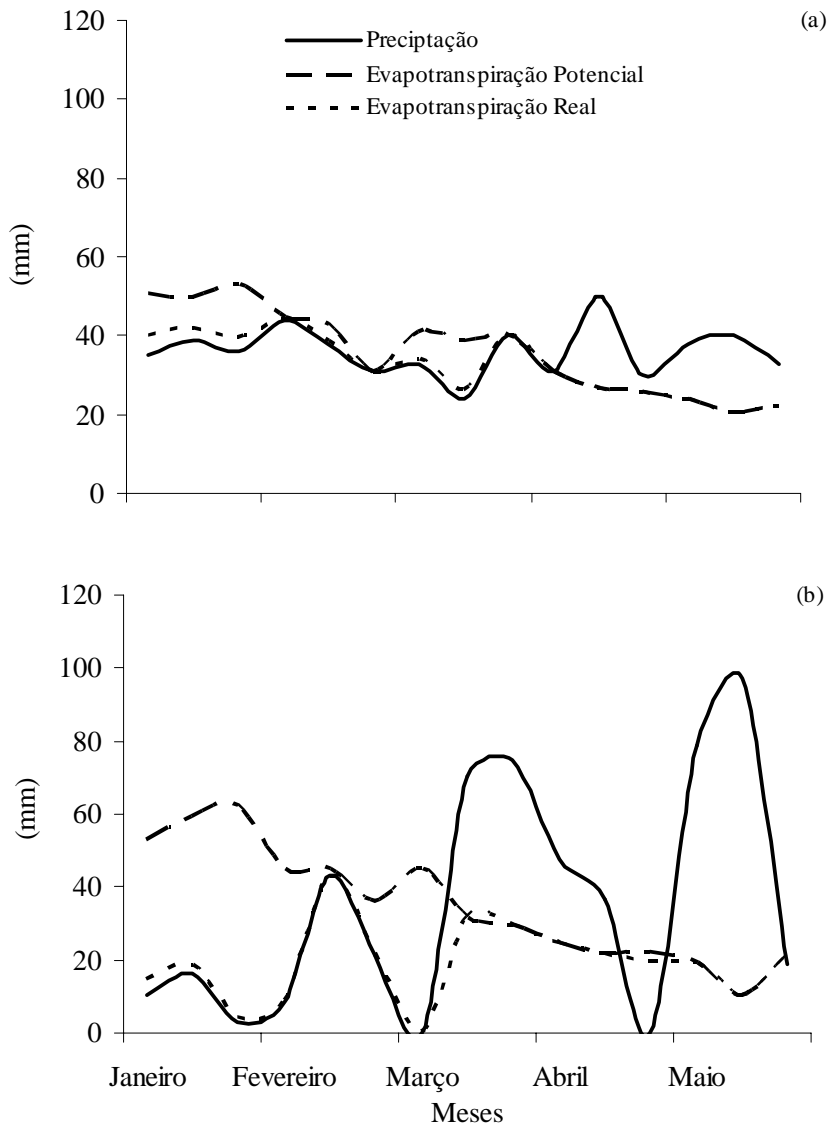

Figura 1 - Balanço hídrico climatológico da Normal - 1969-1999 (a) e do período experimental - 2005 (b).

Tabela 1 - Massa de forragem (MF, kg/ha de MS), massa de forragem verde (MFV, $\mathrm{kg} / \mathrm{ha}$ de MSV) e oferta de forragem (OF, kg MS/100 kg PV/dia) de pastagem nativa submetida à adubação nitrogenada e utilizada por cordeiras

\begin{tabular}{lccc}
\hline Período & MF & MFV & OF \\
\hline $21 / 1-16 / 2$ & $3.515 \mathrm{a}$ & $1.363 \mathrm{a}$ & 15,8 \\
$17 / 2-16 / 3$ & $3.114 \mathrm{~b}$ & $706 \mathrm{~b}$ & 14,6 \\
$17 / 3-13 / 4$ & $2.795 \mathrm{bc}$ & $820 \mathrm{~b}$ & 12,3 \\
$14 / 4-10 / 5$ & $2.464 \mathrm{c}$ & $1.552 \mathrm{a}$ & 13,7 \\
Média & 2.972 & 1.035 & 14,1 \\
\hline CV $(\%)$ & 7,96 & 18,19 & 20,28 \\
\hline P & $<0,0001$ & 0,0002 & 0,2603 \\
\hline
\end{tabular}

a, b, $c$ : Médias seguidas de letras diferentes na coluna diferem $(P<0,1)$ entre si pelo teste Tukey.

portanto, houve provável limitação do consumo, melhor representada pelo modelo de regressão quadrático $\left(\hat{Y}=0,36821 x^{2}-51,66642 x+2.501,71029 ; R^{2}=0,6636 ;\right.$ $\mathrm{P}<0,0001)$, pois a quantidade de material morto foi elevada e, na média dos níveis de nitrogênio, foi igual a $64,7 \%$. A oferta de forragem ficou um pouco abaixo da pretendida, porém, foi possível bom controle desta variável, que foi 
semelhante entre os níveis de nitrogênio $(P=0,6726)$ em todos os períodos $(\mathrm{P}=0,2603)$ e, na média das três doses de nitrogênio, foi igual a $14,1 \%$ e não limitou, em tese, o consumo dos animais. No entanto, a oferta de forragem verde apresentou interação das variáveis dose e período $(\mathrm{P}=0,0613)$. Em virtude do déficit hídrico nos períodos intermediários a OFV apresentou seus menores valores (Figura 2), como consequiênica dos valores elevados de material morto, o que limitou a obtenção pelos animais de uma dieta de qualidade, pois a apreensão de material verde na massa de forragem total foi provavelmente prejudicada. A estrutura do pasto tem importante papel no comportamento do animal em pastejo e, conseqüentemente, nas taxas de ganhos animais (Carvalho, 1997). Segundo Poppi et al. (1987), as mudanças na estrutura alteram o tamanho do bocado, que, por sua vez, é positivamente relacionado à MS, representada pela porção de folhas verdes.

A adubação com $200 \mathrm{~kg} /$ ha de $\mathrm{N}$ resultou em taxa de lotação e carga animal superiores $(\mathrm{P}=0,0110 ; \mathrm{P}=0,0077)$ à área sem aplicação de nitrogênio (Tabela 2), enquanto a aplicação de $100 \mathrm{~kg} / \mathrm{ha}$ de nitrogênio manteve-se em posição intermediária e não diferiu das demais. As variáveis ajustaram-se a um modelo de regressão linear em reposta às doses de nitrogênio (Figura 3), uma vez que cada $100 \mathrm{~kg} / \mathrm{ha}$ de $\mathrm{N}$ proporciona o aumento na LOT de 2,1 animais/ha e de $87,4 \mathrm{~kg} / \mathrm{ha}$ de PV na CA, indicadores do efeito positivo do nitrogênio na pastagem nativa. A LOT e a CA não diferiram entre os períodos de avaliação $(\mathrm{P}=0,1105$; $\mathrm{P}=0,1238$ ), portanto, mesmo em condições totalmente desfavoráveis e decorrentes do déficit hídrico, a pastagem nativa melhorada teve a capacidade de manter altas taxas de lotação, o que só foi possível com a adaptação das espécies nativas às condições edafoclimáticas, o que

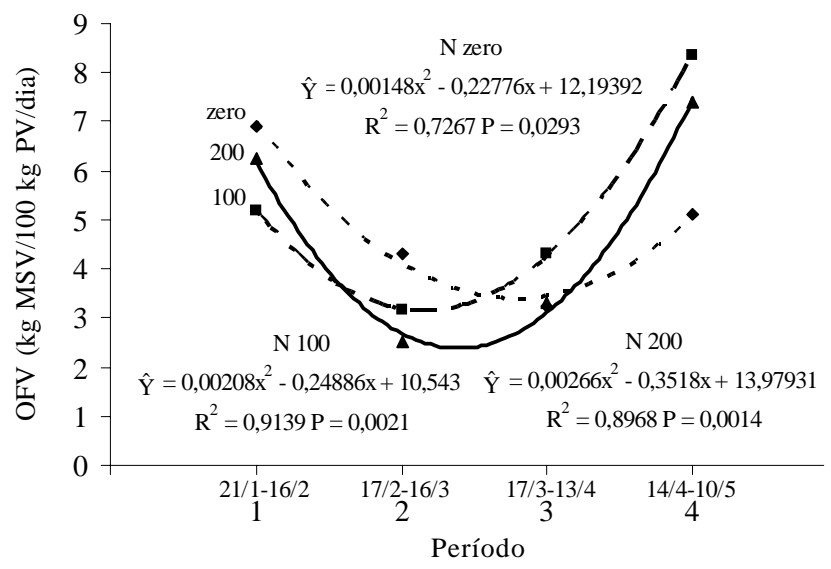

Figura 2 - Oferta de forragem verde (kg MSV/100 kgPV/dia) de pastagem nativa submetida à adubação nitrogenada e utilizada por cordeiras.
Tabela 2 - Taxa de lotação (LOT, animais/ha), carga animal (CA, kg/ha de PV), escore de condição corporal (ECC, 1-5), ganho médio diário (GMD, kg/animal) e ganho de peso por área (GPA, $\mathrm{kg} / \mathrm{ha}$ de $\mathrm{PV}$ ) de cordeiras em pastagem nativa submetida à adubação nitrogenada

\begin{tabular}{lccccc}
\hline $\mathrm{N}(\mathrm{kg} / \mathrm{ha})$ & LOT & CA & ECC & GMD & GPA \\
\hline Zero & $17,8 \mathrm{~b}$ & $689 \mathrm{~b}$ & 2,2 & 0,025 & 44,9 \\
100 & $19,6 \mathrm{ab}$ & $764 \mathrm{ab}$ & 2,1 & 0,024 & 51,4 \\
200 & $22,1 \mathrm{a}$ & $864 \mathrm{a}$ & 2,1 & 0,021 & 41,3 \\
Média & 19,8 & 772 & 2,15 & 0,023 & 45,9 \\
\hline CV $(\%)$ & 11,49 & 11,50 & 5,95 & 10,65 & 16,73 \\
\hline P & 0,0110 & 0,0077 & 0,4805 & 0,9755 & 0,9628 \\
\hline
\end{tabular}

a, b: Médias seguidas de letras diferentes na coluna diferem $(P<0,1)$ entre si pelo teste Tukey.

provavelmente não seria possível com espécies cultivadas exóticas, que não têm este potencial de adaptação.

O desaparecimento de MS não diferiu entre os níveis de nitrogênio $(P=0,8649)$ em todos os períodos $(P=0,4184)$ e, na média, foi igual a $2,9 \%$. Em baixos valores de oferta de forragem, o DPV corresponde ao que foi consumido pelos animais, mas, à medida que a oferta de forragem aumenta, a fração consumida se diferencia mais do desaparecimento, uma vez que a fração perdida por senescência e pisoteio aumenta (Boggiano, 2000). O potencial de consumo destes animaisé de 3,5\% PV (NRC, 1985), e o valor de DPV foi inferior. Essa variável contempla consumo, senescência e outras "perdas" e indica que os animais tiveram restrição ao consumo de forragem. Conforme estudos de Gibb \& Treacher (1976), para que a ingestão de MS não esteja limitada, deve-se ofertar três vezes o potencial de consumo. Possíveis restrições, neste caso, devem estar relacionadas à disponibilidade de material verde no pasto, e não à quantidade de forragem residual total.

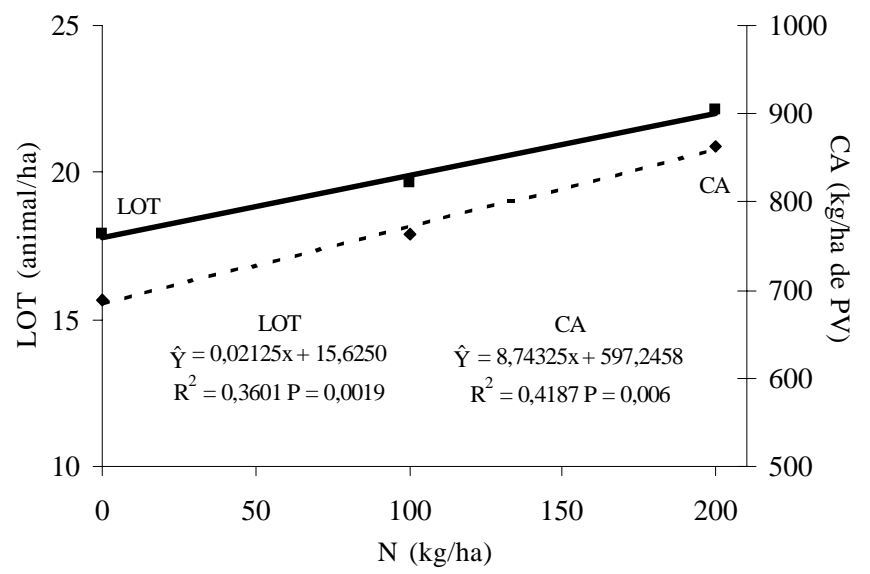

Figura 3 - Taxa de lotação (LOT, animais/ha) e carga animal (CA, kg/ha de PV) suportada pela pastagem nativa submetida à adubação nitrogenada e utilizada por cordeiras. 
Conseqüentemente, o ganho médio diário não diferiu $(\mathrm{P}=0,9755)$ entre as doses de nitrogênio e, na média, foi igual a $0,023 \mathrm{~kg} / \mathrm{animal}$ (Tabela 2). Os valores desta variável oscilaram no decorrer do período de avaliação $(\mathrm{P}=0,0268)$ e este comportamento na média das doses de nitrogênio foi melhor representado por modelo de regressão quadrático. Esta variável apresenta o mesmo comportamento da OFV (Figura 2) e permite atribuir os baixos desempenhos individuais à qualidade da forragem disponível consumida, uma vez que a oferta média de forragem verde situou-se em 5\%. O coeficiente de correlação de Pearson $(r)$ de $0,65(P=0,0006)$ demonstra uma relação consistente entre OFV e GMD. Além disso, esses ganhos ficaram bem abaixo da expectativa, provavelmente em razão da categoria animal, que é muito exigente, com forte demanda para o crescimento e também pelas condições climáticas não satisfatórias. Apesar de não ter ocorrido diferenças significativas entre os níveis de nitrogênio nos diferentes períodos, a ausência de adubação $(0 \mathrm{~kg} / \mathrm{ha}$ de $\mathrm{N})$ no primeiro período resultou em maiores GMD, o que é explicado pelo maior valor de OFV obtido neste período. No entanto, no último período, houve tendência de maiores GMD, como resultado dos maiores valores de OFV e da maior taxa de acúmulo de forragem, decorrente do maior nível de reservas nitrogenadas com a aplicação de nitrogênio (Lemaire \& Millard, 1999) e da segunda aplicação de nitrogênio ao início do terceiro período, conjuntamente à ocorrência de precipitações.

O escore de condição corporal não diferiu $(\mathrm{P}=0,4805)$ entre os níveis de adubação nitrogenada (Tabela 2) e ajustou-se ao modelo de regressão quadrático $\left(\hat{Y}=0,0001481 x^{2}-0,02371 x+2,94719 ; R^{2}=0,6518 ; P=0,0001\right)$,

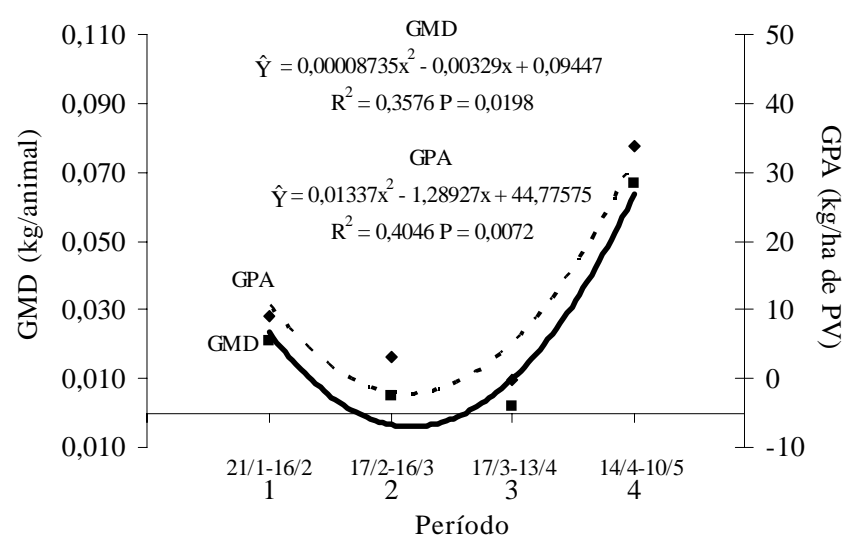

Figura 4 - Ganho médio diário (GMD, kg/animal) de cordeiras e ganho de peso por área (GPA, kg/ha de PV) em pastagem nativa submetida à adubação nitrogenada. com pequena variação durante os períodos de avaliação $(\mathrm{P}=0,0010)$, cujos valores médios foram 2,$4 ; 2,1 ; 2,0 \mathrm{e} 2,2$ nos períodos de avaliação 1, 2, 3 e 4, respectivamente. Embora o ECC tenha apresentado baixa variação, apresenta tendência semelhante à OFV e GMD. Esses valores baixos são decorrentes do déficit hídrico, que causou OFV limitante para o consumo dos animais (Figura 2). Pereira Neto (2004) indica que o ECC de 3,0-3,5 para o período de acasalamento, mas Gunn et al. (1984) indica 2,5 como crítico mínimo para se obterem taxas de ovulação satisfatórias. Os animais apresentaram ECC abaixo do recomendado para o período de reprodução, pois, mesmo com a ocorrência de precipitações nos últimos períodos, não houve tempo suficiente para os animais melhorarem seus ECC.

O ganho de peso por área não diferiu $(\mathrm{P}=0,9628)$ entre os níveis de nitrogênio e, na média, foi igual a $45,9 \mathrm{~kg} / \mathrm{ha} \mathrm{de}$ PV (Tabela 2) e oscilou no decorrer do período de avaliação $(\mathrm{P}=0,0361)$ ajustando-se a um modelo de regressão quadrático. Como conseqüência dos menores GMD nos períodos de maior déficit hídrico explicado anteriormente, o GPA apresentou resposta semelhante da OFV e do GMD (Figura 4), com valores baixos e até mesmo negativos nos períodos intermediários, nos quais o déficit hídrico foi mais severo. Nesta variável, assim com no GMD, a área sem aplicação de nitrogênio também apresentou tendência de maiores valores em comparação à aplicação de nitrogênio no período inicial. No período final, com o retorno das precipitações, verificou-se comportamento inverso, de modo que as doses 100 e $200 \mathrm{~kg} / \mathrm{ha}$ de nitrogênio resultaram em valores superiores, fato atribuído às maiores OFV, que proporcionaram aos animais dietas de maior valor nutritivo, e que resultou em maiores GMD e GPA, uma vez que o GMD é uma das variáveis que compõem o GPA.

No período de acasalamento, a MF apresentou valor médio de $2.115 \mathrm{~kg} / \mathrm{ha}$ de $\mathrm{MS}$, inferior $(\mathrm{P}=0,0008)$ ao do préacasalamento ( $2.972 \mathrm{~kg} / \mathrm{ha}$ de MS), em razão do aumento da LOT, que passou de 19,8 para 22 animais/ha $(\mathrm{P}=0,0856)$, fato explicado pela entrada dos carneiros nos piquetes no período de monta. A MFV, no entanto, apresentou resposta inversa em MFe aumentou de 1.035 para $1.206 \mathrm{~kg} / \mathrm{ha} \mathrm{de} \mathrm{MS}$ no período de reprodução $(\mathrm{P}<0,1)$, que foi proporcionado pela não ocorrência de déficit hídrico neste período. Essa resposta influenciou diretamente a OFV, que também teve resposta positiva e aumentou de 5 para $8 \%(\mathrm{P}=0,0008)$. A oferta de forragem média das doses de nitrogênio manteve-se semelhante ao período anterior $(\mathrm{P}=0,7871)$, com valor médio de $14,4 \%$. Durante este período, os valores de GMD foram inferiores aos do pré-acasalamento $(\mathrm{P}<0,0001)$, 
média -0,078 kg/animal, provavelmente em decorrência do estresse do acasalamento.

Durante o período de acasalamento, utilizou-se a técnica de sincronização de estro, que é importante e indispensável quando são empregados sistemas intensivos de reprodução, como parições em blocos ou três partos a cada dois anos (Moraes et al., 2002). A utilização de prostaglandina ( $\mathrm{PG}_{2} \alpha$, Dinoprost Trometamina) para sincronização de estros deve ser utilizada somente durante o período reprodutivo e tem como fator limitante que os animais estejam ciclando e apresentem corpo lúteo funcional, ou seja, entre os dias 5 a 14 do ciclo (Hoppe \& Slyter, 1989).

A sincronização de estro não diferiu entre os níveis de adubação nitrogenada ( $\mathrm{P}=0,6247)$. Na primeira sincronização, $89,7 \%$ das cordeiras manifestaram estro e a média total de cada tratamento foi de 92,9,92,3 e 100\%, para 0,100 e $200 \mathrm{~kg} / \mathrm{ha}$ de nitrogênio, respectivamente, considerando como média das três doses $94,9 \%$ de manifestação de estro. A sincronização é uma técnica que ajuda na organização dos sistemas de produção melhorando o rendimento do rebanho. No entanto, a técnica não aumenta a fertilidade do rebanho; apenas organiza a produção das fêmeas e torna possível incrementar a produção por organizar o sistema de parição. As técnicas de sincronização de cio sincronizam o período dos partos, permitindo oferecer melhores condições alimentares quando as fêmeas requerem maior quantidade de alimento.

A taxa de prenhez apresentou resposta semelhante à sincronização e não diferiu entre as doses de nitrogênio ( $P=0,6337)$, em média, 53,2\%. Esse valor moderado pode ser explicado pelo fato de, em animais jovens, os sinais comportamentais do estro ser usualmente fracos com intensidade menos marcante que em ovelhas adultas. Além disso, a duração do estro é mais curta e o número de ciclos estrais é menor que o de ovelhas durante a estação reprodutiva (Dyrmundsson \& Hallgrãsson, 1978).

Sá et al. (1998), acasalando cordeiras com idade, peso e ECC médios de 8 meses, 37,4 kg e 3,3 no início estação de monta, respectivamente, apresentaram taxa de prenhez de 61,2\%. Ducker \& Boyd (1977) relatam que, ao mesmo peso corpóreo, ovelhas de pequeno porte e alto ECC possuem maior taxa de ovulação que ovelhas maiores com baixo ECC, o que justifica a importância desta variável, que é reflexo do estado nutricional dos animais e função direta do GMD. No início do período de acasalamento, as cordeiras tinham, em média, 10 meses, 39,2 kg de peso e 2,1 de ECC.

Outro fator que influenciou o moderado desempenho reprodutivo foi a duração da estação de monta, de apenas 15 dias, pois não foi possível prolongar este período, uma vez que houve aumento da LOT com a inclusão dos carneiros e as condições do pasto não permitiram a continuidade das avaliações. Para que isto fosse possível, seria necessário diminuir o número de animais-teste dos piquetes.

\section{Conclusões}

A diminuição da oferta de forragem verde da pastagem nativa a níveis limitantes para o consumo, em condições de déficit hídrico, limita o desenvolvimento de cordeiras e seu posterior desempenho reprodutivo, uma vez que a adubação nitrogenada não contribui para atenuar estes efeitos. Há efeito positivo da adubação nitrogenada sobre a taxa de lotação, mas a resposta é de baixa magnitude quando ocorre limitação hídrica. A utilização da oferta de massa total como critério de manejo com vistas à produção animal não é adequada quando a proporção de material morto é elevada. Não se justifica a aplicação de nitrogênio em condições ambientais desfavoráveis, pois os custos de produção aumentam e a adubação não proporciona vantagens ao sistema. Com a intensificação, a interdependência entre o componente econômico e a resposta biológica aumenta.

\section{Agradecimento}

Ao Conselho Nacional de Desenvolvimento Científico e Tecnológico (CNPq), pela concessão de bolsas. À Empresa Agropecuária Cerro Coroado, pela disponibilização dos animais. Aos integrantes do Grupo de Pesquisa em Ecologia do Pastejo pelo auxílio na execução das avaliações de campo.

\section{Literatura Citada}

BARCELlos, J.M.; SEVERO, H.C.; ACEVEDO, A.S. et al. Influência da adubação e sistemas de pastejo na produção de pastagens naturais. Bagé: CNPO/EMBRAPA, 1987. v.1, p.11-16. (Coletâneas das pesquisas forrageiras).

BECK, N.F.G.; DAVIES, M.C.G. The effect of stage of breeding season or pre-mating oestrogen and progestagen therapy on fertility in ewe lambs. Animal Production, v.59, p.429-434, 1994.

BECK, N.F.G.; DAVIES, M.C.G; DAVIES, B. A comparison of ovulation rate and late embryonic mortality in ewe lambs and ewes and the role of late embryo loss in ewe lamb subfertility. Aimal Science, v.62, p.79-83, 1996.

BEMHAJA, M.; BERRETA, E.J.; BRITO, G. Respuesta a la fertilización nitrogenada de campo natural en basalto profundo. In: REUNIÓN DEL GRUPO TÉCNICO REGIONAL DEL CONE SUR EN MEJORAMIENTO Y UTILIZACIÓN DE LOS RECURSOS FORRAJEROS DEL ÁREA TROPICAL Y SUBTROPICAL: GRUPO CAMPOS, 14., 1994, Termas de Arapey. Anais... Montevideo: INIA, 1998. p.119-122. (Série Técnica, 94).

BERGAMASCHI, H.; GUADAGNIN, M.R.; CARDOSO, L.S. et al. Clima da Estação Experimental da UFRGS (e Região de Abrangência). Porto Alegre: Universidade Federal do Rio Grande do Sul, 2003. 78p. 
BOGGIANO, P.R.; MARASCHIN, G.E.; NABINGER, C. et al. Efeito da adubação nitrogenada e oferta de forragem sobre as taxas de acúmulo de matéria seca numa pastagem nativa do Rio Grande do Sul. In: REUNIÃO DO GRUPO TÉCNICO EM FORRAGEIRAS DO CONE SUL - ZONA CAMPOS, 18., 2000, Anais... Guarapuava, 2000. p.120-121.

CARVALHO, P.C.F. A estrutura da pastagem e o comportamento ingestivo de ruminantes em pastejo. In: SIMPÓSIO SOBRE AVALIAÇÃO DE PASTAGENS COM ANIMAIS, 1997, Maringá. Anais... Maringá: Universidade Estadual de Maringá, 1997. p. 25-52.

DAVIES, M.C.G.; BECK, N.F.G. A comparison of plasma prolactin, $\mathrm{LH}$ and progesterone concentrations during oestrus and early pregnancy in ewe lambs and ewes. Animal Production, v.57, p. 281-286, 1993

DUCKER, M.J.; BOYD, J.S. The effect of body size and body condition on the ovulation rate of ewes. Animal Production, v.24, p.377-385, 1977.

DYRMUNDSSON, O.R.; HALLGRÃNSSON, S. Reproductive efficiency of Iceland sheep. Livestock Production Science, v.5, p.231-234, 1978 .

EMPRESA BRASILEIRA DE PESQUISA AGROPECUÁRIA EMBRAPA. Centro Nacional de Pesquisa do Solo - CNPS. Sistema Brasileiro de Classificação de Solos. Rio de Janeiro: Embrapa, 1999. 412p.

GIBB, M.J.; TREACHER T.T. The effect of herbage allowance on herbage intake and performance of lambs grazing perennial ryegrass and red clover swards. Journal of Agricultural Science, v.86, p.355-365, 1976

GOMES, L.H. Produtividade de um campo nativo melhorado submetido à adubação nitrogenada. Porto Alegre: Universidade Federal do Rio Grande do Sul, 2000. 124p. Dissertação (Mestrado em Zootecnia) - Universidade Federal do Rio Grande do Sul, 2000.

GUMA, J.M.C.R. Parâmetros da pastagem e produção animal em campo nativo adubado e fertilizado com diferentes doses de nitrogênio, submetido para diferimento para utilização no outono-inverno. Porto Alegre: Universidade Federal do Rio Grande do Sul, 2005. 58p. Dissertação (Mestrado em Zootecnia) Universidade Federal do Rio Grande do Sul, 2005.

GUNN, R.G.; DONEY, J.M.; SMITH, W.F. The effect of level of pre-mating nutrition on ovulatory rate in Scottish blackface ewes in different body conditions at mating. Animal Production, v.39, p.235-239, 1984.

HAYDOCK, K.P.; SHAW, N.H. The comparative yield method for estimating dry matter yield of pasture. Australian Journal of Agriculture and Animal Husbandry, v. 15 , p.66-70, 1975.

HOPPE, K.F.; SLYTER, A.L. Effects of prostaglandin dosage on synchronizing ovine estrous using a modified single injection regimen. Theriogenology, v.31, p.1191-1200, 1989.

LEMAIRE, G.; MILLARD, P. An ecophysiological approach to modeling resource fluxes in competing plants. Journal of Experimental Botany, v.50, p.15-28, 1999.

LEWIS, R.M.; NOTTER, D.R.; HOGUE, D.E. et al. Ewe fertility in the STAR accelerated lambing system. Journal of Animal Science, v.74, p.1511-1522, 1996.
MORAES, A.; MOOJEN, E.L.; MARASCHIN, G.E. Comparação de métodos de estimativa de taxa de crescimento em uma pastagem submetida a diferentes pressões de pastejo. In: REUNIÃO ANUAL DA SOCIEDADE BRASILEIRA DE ZOOTECNIA, 27. 1990, Campinas. Anais... Piracicaba: Fundação de Estudos Agrários Luiz de Queiroz, 1990. p.332.

MORAES, J.C.F.; SOUZA, C.J.H.; GONÇALVES, P.B.D. Controle do estro e da ovulação em bovinos e ovinos. In: GONÇALVES, P.B.D.; FIGUEIREDO, J.R.; FREITAS, V.J.F. (Eds.) Biotécnicas aplicadas à reprodução animal. São Paulo: Varela Editora e Livraria Ltda, 2002. p.25-55.

MOREnO, J.A. Clima do Rio Grande do Sul. Porto Alegre: Secretaria da Agricultura, 1961. 41p.

MOTT, G.O., LUCAS, H.L. The design conduct and interpretation of grazing trials on cultivated and improved pastures. In: INTERNATIONAL GRASSLAND CONGRESS, 6., 1952, Pensylvania. Proceedings... Pensylvania: State College Press, 1952. p.1380-1395.

NATIONAL RESEARCH COUNCIL - NRC. Nutrient requirements of sheep. 6.ed. Washington D.C.: National Academy Press, 1985. 90p.

PEREIRA NETO, O.A. Escore de condição corporal. Instrumento de tomada de decisão. In: PEREIRA NETO, O.A.; MÓRLAN, J.B.; CARVALHO, P.C.F. et al. (Eds.) Práticas em Ovinocultura - Ferramentas para o sucesso. Porto Alegre: SENAR, 2004. p.67-78.

POPPI, D.P.; HUGHES, T.P.; L'HUILLIER, P.J. Intake of pasture by grazing ruminants. In: NICOL, A.M. (Ed.) Feeding livestock on pasture. Hamilton: New Zealand Society of Animal Production, 1987. p.55-63.

POWELL, M.R.; KAPS, M.; LAMBERSON, W.R. et al. Use of melengestrol acetate-based treatments to induce and synchronize estrus in seasonally anestrous ewes. Journal of Animal Science, v.74, p.2292-2302, 1996.

RIBEIRO, L.A.O.; GREGORY, R.M.; MATTOS, R.C. Prenhez em rebanhos ovinos do Rio Grande do Sul. Ciência Rural, v.32, n.4, p.637-641, 2002.

RODA, D.S.; SANTOS, L.E.; CUNHA, E.A. et al. Performance of ewes mated at intervals of eight months. Boletim da Industria Animal, v.50, p.49-54, 1993.

ROSITO, J.M. Levantamento fitossociológico de uma pastagem perene de verão, submetida a diferentes sistemas de manejo. Porto Alegre: Universidade Federal do Rio Grande do Sul, 1983. 181p. Dissertação (Mestrado em Zootecnia) Universidade Federal do Rio Grande do Sul, 2000.

RUSSEL, A. Body condition scoring of sheep. In: BODEN, E. (Ed.) Sheep and goat practice. London: Bailliere Tindall, 1991. p.3-10.

SA, J.L.; OTTO, C.; ANDRIGUETTO, J.L. et al. Efeito da antecipação reprodutiva no desempenho de borregas. In: REUNIÃO ANUAL DA SOCIEDADE BRASILEIRA DE ZooteCniA, 35., 1998, Botucatu. Anais... Botucatu: Sociedade Brasileira de Zootecnia, 1998. p.160-162.

STATISTICAL ANALYSES SYSTEM - SAS. SAS/STAT user's guide: version 6.12. Cary: SAS Institute, 1997. 1167p.

THORNTHWAITE, C.W.; MATHER, J.R. The water balance. Washington: Drexel Institute of Technology, 1955. 104p. 